\title{
Images of gas molecules by electron holography. I. Theory
}

\author{
L. S. Bartell \\ Department of Chemistry, The University of Michigan, Ann Arbor, Michigan 48109 \\ (Received 26 October 1978) \\ It is demonstrated that molecular images with an Abbe resolution limit as fine as $0.1 \AA$ can, in principle, \\ be reconstructed optically directly from appropriately recorded electron diffraction plates (holograms). \\ Even when a sample of randomly oriented gas-phase molecules is chosen as a subject, reconstructed \\ images with a well-defined physical interpretation can be made. If the molecules are selected to contain \\ just one heavy atom-this atom to generate a strong "reference wave," by scattering, so as to satisfy \\ Gabor's conditions for the production of a hologram-the reconstructed images correspond to rotational \\ averages about the heavy atom. If no single atom dominates, the "image" is not as simple conceptually \\ but the reconstruction is shown to display a series of rings, each of which corresponds to an interatomic \\ distance in the molecule. The physical basis of the approach is reviewed, followed by a mathematical \\ treatment of the electron scattered intensity and optical reconstruction.
}

\section{INTRODUCTION}

In the half-century since its inception, ${ }^{1}$ vapor-phase electron diffraction has yielded a rich store of molecular information. Virtually all of this information has been derived from photographically recorded diffraction patterns by digital computations based on measurements of positions and intensities of diffraction features. It escapted notice that such information can, in principle, be extracted quite directly from the diffraction plates by optical reconstruction. Indeed, if certain conditions could be met, three-dimensional molecular images would be apparent in optical interference patterns made from electron diffraction plates.

Even in the case of conventional experimental condi tions, the Fraunhofer diffraction patterns produced by passing monochromatic light through electron diffraction patterns exhibit maxima related to atomic positions. Once noted, this fact is immediately evident from elementary mathematical considerations without special reference to the theory of image formation. This fact was only recognized, however, after comparatively recent investigations of alternative ways to apply Gabor's 1948 holographic theory of image reconstruction. ${ }^{2}$ Gabor's work had been stimulated by Bragg's ${ }^{3}$ and Buerger's ${ }^{4}$ ingenious " $x$-ray microscopes" devised to form images of molecules from digital data by photographic or optical Fourier techniques. These advances, in turn, had been inspired by Abbe's 1873 treatment of image formation. ${ }^{5}$

Several years ago, enormously magnified images of gas-phase atoms, with resolution limits below $0.1 \AA$, were generated from electron diffraction patterns taken to comply with holographic requirements. ${ }^{6,7}$ For technical reasons, it is simpler to reconstruct facsimilies of the electron clouds in monatomic elements than to reproduce interatomic details in polyatomic molecules. Subsequently, an optical filter was devised to discriminate against obtrusive instrumental artifacts and permit the photographing of holographic images of molecules. The first successful pictures ${ }^{8}$ were of images of $\mathbf{A s F}_{5}$, rotationally averaged about the central arsenic atom. A physical explanation of the method is outlined in the next section and followed by a mathematical treatment.

\section{PHYSICAL BASIS OF METHOD}

\section{A. Image formation}

Before deriving quantitative expressions relating image intensities to molecular and instrumental parameters it is helpful to consider the type of information derivable by the present two-stage procedure. In the first stage, electron waves are scattered by a subject (say, a molecule). The scattered amplitude in the resultant Fraunhofer diffraction pattern corresponds to a Fourier transform of the scattering potential in the subject. It it were possible to record this scattered amplitude on a photographic plate, the developed plate, displaying the scattered subject-waves as modulations in absorbance, could act in the second stage of the procedure as a diffraction grating ("Fourier-transform hologram") 9 to a beam of visible light. The optical Fraunhofer diffraction pattern, so obtained, would represent a Fourier transform of a Fourier transform and would thereby restore the form of the original subject. Such a procedure is the essence of holography as conceived of by Gabor. ${ }^{2}$ Because no lens is required between the subject and the photographic plate (hologram), the scheme should, in principle, be able to produce images displaying a greater resolving power than is attainable by direct microscopy utilizing the electron lenses of small numerical aperture now available.

Gabor showed that it is entirely possible to record the scattered amplitudes, rather than simply their square (the intensity) if the subject waves are coherently superposed upon a reference wave dominating the amplitude received by the photoplate. His proposal that the divergent reference wave be generated from the incident beam before it encounters the subject proved to work very well in subsequent optical holographic studies. Unfortunately, it has been less effective in electron-wave holography. An alternative scheme for electrons, namely, the generation of a reference wave by a strong scattering of the incident beam within the subject itself (say, by a heavy atom in the molecules under scrutiny) has been much more successful. ${ }^{6-8}$ In this alternative scheme, even gas-phase molecules can serve as subjects because each one, producing its own reference wave internally, generates its own hologram. 


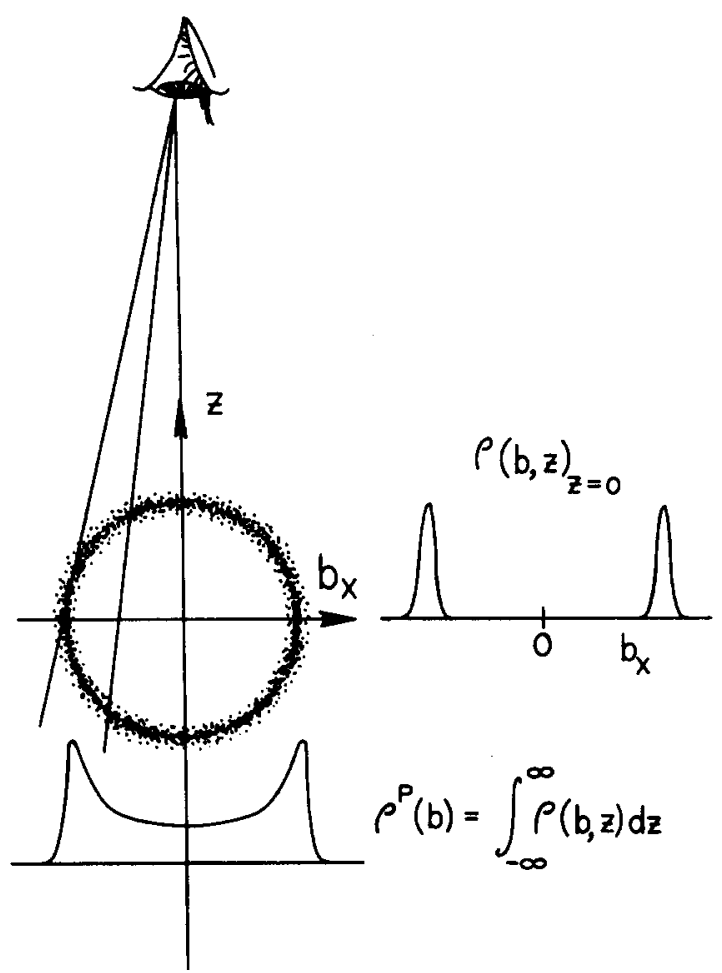

FIG. 1. Schematic illustration of projected density $\rho^{P}(b)$ corresponding to image of spherical average distribution of atom $i$ around a heavy central atom $n$.

Since holograms of all molecules, each recorded at the Fraunhofer limit, fall exactly on top of each other, the reconstruction is an ensemble of images, each with the heavy atom at the origin. An elementary pictorial explanation of the physical basis of the method is given elsewhere. ${ }^{?}$

If it were possible to have a sample of perfectly oriented gas-phase molecules, the resultant image would be of an oriented molecule (together with a twin image corresponding to an inversion through the heavy atom origin). In the real case of gas-phase molecules with chaotic orientations, the image is a spherical average about the heavy atom. Internuclear distances from the heavy atom show up as rings of high image intensity. Measurements of the ring radii give the distances directly. If no single atom dominates the scattering, the "image" corresponds to rings representing intramolecular distances reckoned from each atom, each atom successively being placed at the origin. What is seen is closely related to the spherical average of the Patterson function of the molecules. ${ }^{10}$

It is worthwhile to review certain practical limitations governing the appearance of the final image. First, because the resolving power parallel to the optic axis of the system is considerably poorer than that perpendicular to the axis, the depth of focus is comparable to or greater than the thickness of the simple molecules studied to date. Therefore, a molecule of $\mathrm{AsF}_{5}$, for example, would not be seen as a spherical shell of fluorines around the heavy atom but, rather, is the projection of a spherical shell on the image plane. That is, what is seen along a given line of sight is the sum total of rotationally averaged molecular matter from the back to the front, as is illustrated in Fig. 1. Since this projected image intensity is finite inside the actual shell radius but zero outside, the internuclear distance peaks are skewed inwards and perceived as being somewhat smaller in radius than the true interatomic distances. The shortening to be expected can be calculated readily, as shown in the next paper (Paper II). ${ }^{11}$ In the case of more complex gas-phase molecules, the image is a superposition of projections of spherical shells.

Secondly, the method is practical only if an appropriate "filter" (rotating sector) is used in the recording of the electron hologram. Since this filter necessarily has a small angle cutoff as well as a large angle cutoff, a significant signal distortion is inescapable.

This and other influences of finite numerical aperture, finite wavelength, and first-and second-stage filters are treated in the following paper. ${ }^{11}$

\section{B. Resolving power and depth of focus}

As discussed in more detail elsewhere, ${ }^{7}$ the resolving power attainable in this variant of holography is very nearly that of standard microscopes operating with the same wavelength, $\lambda_{1}$, and numerical aperture, $\sin \theta_{m}$ (see Fig. 2). Denoting the minimum resolvable separation as $\epsilon$, we write, for the Abbe limit of resolution, $\epsilon_{p}$, perpendicular to the optic axis

$$
\epsilon_{p} \approx \lambda_{1} / 2 \sin \theta_{m} \text {. }
$$

Parallel to the optic axis, the limiting resolution $\epsilon_{a x}$, or depth of focus, can be expressed as

$$
\epsilon_{a x} \approx 2 \lambda_{1} / \sin ^{2} \theta_{m} \text {. }
$$

Somewhat more accurate expressions taking into ac count the fact that the reconstructions involve coherent imaging are given in Ref. 7. Representative operating conditions in the experiments to be described, with $\lambda_{1}$ $\approx 0.06 \AA$ and $\sin \theta_{m} \approx 0.20$, lead to the values $\epsilon_{p} \approx 0.15 \AA$ and $\epsilon_{a x} \approx 3 \AA$.
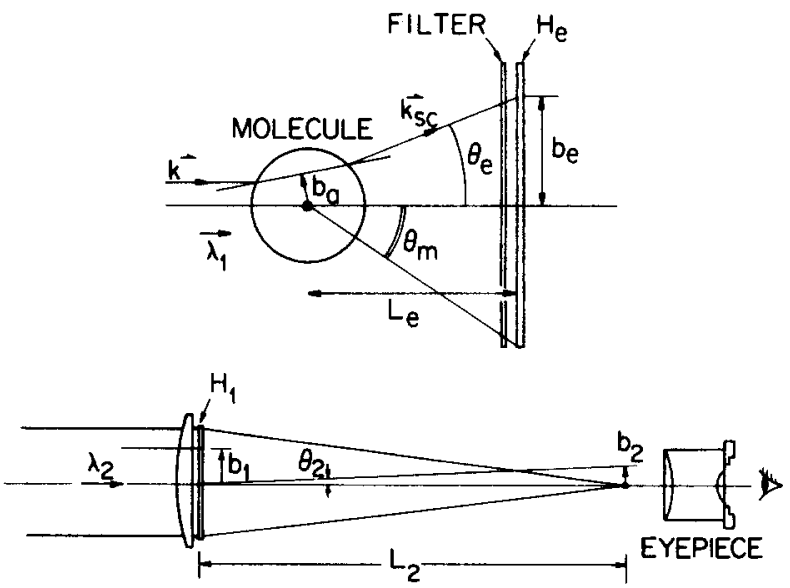

FIG. 2. Schematic diagram of the two stages of a holographic microscope. Upper figure: Production of a hologram $H_{\theta}$ by means of electron waves. Lower figure: Reconstruction of image by transmission of light through hologram $H_{1}$. $H_{1}$ is a reduced copy of $H_{e}$ on an optically perfect plate. 


\section{MATHEMATICAL TREATMENT}

\section{A. Specification of system}

Figure 2 displays schematically the essential components of the two stages of the holographic microscope and labels the principal geometric variables. In stage 1, a hologram $H_{e}$ is formed by electron waves of wavelength $\lambda_{1}$. Geometric variables characterizing the scat tering angle $\theta=\arctan (b / L)$, where $b$ is a radial vector in the plane of the hologram, are subscripted with letter $e$ in the first, electron-wave stage and with the number 1 in the corresponding left-hand region of stage 2. Note that wavelengths $\lambda_{2}$ and $\lambda_{1}$ are enormously different and that, in our current practice, $H_{1}$ is considerably re duced in size from $H_{e}$. Our holograms and reconstructions have axial symmetry consistent with the spherical symmetry of the rotating molecular subjects.

\section{B. Diffraction of electron wave}

For electrons accelerated through a potential difference of $40-60 \mathrm{kV}$, a "semikinematic" approximation has been observed to hold with an accuracy adequate for the present purposes for atoms with atomic numbers up to 70 or more. ${ }^{12}$ According to this approximation, each atom $i$ at a position $\mathbf{r}_{i}$ in a molecule scatters a wave with amplitude $f_{i}\left(s_{e}\right) \exp \left(i s_{e} \cdot r_{i}\right)$, just as it would in the kinematic (Born) approximation, except that the scattering factor $f_{i}\left(s_{e}\right)$ must be taken as a complex factor $\left|f_{i}\right| \exp i \eta_{i}$ instead of the simple Born factor (which is real). At the aforementioned voltages, however, the absolute magnitude $\left|f_{1}\right|$ does not differ greatly from the Born value $\left(2 / a_{0}\right)\left[Z-F\left(s_{e}\right)\right]_{i} / s_{e}^{2}$ in which $Z$ and $F\left(s_{e}\right)$ represent the atomic number and $\mathrm{x}$-ray scattering factor, $a_{0}$ is the Bohr radius $\hbar^{2}\left(1-v^{2} / c^{2}\right)^{1 / 2} / m_{0} e^{2}$, and $s_{e}$ is $\left(4 \pi / \lambda_{1}\right) \sin \left(\theta_{e} / 2\right)$. Accordingly, the intensity scat tered by an ensemble of gas molecules and reaching the flat photographic plate $H_{e}$ after being filtered by the rotating sector is

$$
\begin{aligned}
I\left(b_{e}\right)= & K_{e} \Phi \cos ^{3} \theta_{e}\left\{\sum_{i}\left[\left|f_{i}\right|^{2}+\left(2 / a_{0} s_{e}^{2}\right)^{2} S_{i}\left(s_{e}\right)\right]\right. \\
& +2 \sum_{i<j}\left|f_{i}\right|\left|f_{i}\right| \cos \left(\eta_{i}-\eta_{j}\right) \int P_{i j}(r) \\
& \left.\times\left(\sin _{e} r\right) / s_{e} r d r\right\},
\end{aligned}
$$

where $K_{e}$ is a collection of factors of no concern here, $\Phi\left(b_{e}\right)$ expresses the effect of the rotating sector, $S_{i}\left(s_{e}\right)$ is the inelastic scattering factor of atom $i$, and $P_{i j}(r) d r$ is the probability that the separation of atoms $i$ and $j$ is between $r$ and $r+d r$. It is apparent that the intensity can be expressed as

$$
I\left(b_{e}\right)=I_{\mathrm{at}}+I_{\mathrm{mol}},
$$

a sum of atomic (single summation) and molecular (double summation) terms. It is useful to reexpress this as

$$
I\left(b_{e}\right)=I_{\mathrm{at}}\left[1+M\left(s_{e}\right)\right],
$$

where $M\left(s_{e}\right)=I_{\mathrm{mol}} / I_{\mathrm{at}}$ is a "reduced molecular intensity" given by

$$
M\left(s_{e}\right)=2 \sum_{i<j} \sum_{i j} \int P_{i j}(r)\left(\sin s_{e} r\right) / s_{e} r d r
$$

in which the coefficients

$$
A_{i j}=\frac{\left|f_{i}\right|\left|f_{j}\right| \cos \left(\eta_{i}-\eta_{j}\right)}{\left\{\sum_{k}\left[\left|f_{k}\right|^{2}+\left(2 / a_{0} s_{e}^{2}\right)^{2} S_{k}\left(s_{e}\right)\right]\right\}}
$$

are approximately proportional to $Z_{i} Z_{j}$ and nearly constant, except at very small $s_{e}$, if the atomic numbers are not large $(Z<35$ or so for $40 \mathrm{kV}$ electrons).

Since $P_{1 j}(r)$ represents the radial distribution $4 \pi r^{2} \rho_{i j}(r)$ of atom $i$ relative to atom $j$, and $\rho_{i j}(r)$ represents the spherically averaged density, the projected density $\rho_{i j}^{P}\left(b_{a}\right)$ of the spherical shell (ideal image). as a function of the impact parameter $b_{a}$ is

$$
\begin{aligned}
\rho_{i j}^{P}\left(b_{a}\right) & =\int_{-\infty}^{\infty} \rho_{i j}(r) d z \\
& =2 \int_{b_{a}}^{\infty} \rho_{i j}\left(b_{a}, z\right)\left[r^{2}-b_{a}^{2}\right]^{-1} d r,
\end{aligned}
$$

where $z$ is the direction along the line of sight conveniently taken ${ }^{13}$ as parallel to $\left(k_{0}+k_{s c}\right) / 2$, the mean of the incident and scattered wave vector directions, and where

$$
r^{2}=b_{a}^{2}+z^{2} \text {. }
$$

The key identity needed in the treatment is

$$
\begin{aligned}
\int P_{i j}(r)\left(\operatorname{sins}_{e} r\right) / s_{e} r d r & =\int \exp \left(i \mathbf{s}_{e} \cdot \mathbf{r}\right) \rho_{i j}(r) d^{3} r \\
& =\int \exp \left(i \mathbf{s}_{e} \cdot \mathbf{b}_{a}\right) \rho_{i j}^{P} d^{2} b_{a} .
\end{aligned}
$$

This term transforms the molecular intensity $I_{\text {mol }}$ into a form readily susceptible of holographic interpretation.

In holographic studies designed to reveal the distribution of electron density in atoms, it is necessary to use a filter function $\Phi$ fashioned to make the coefficient $\Phi \cos ^{3} \theta_{e} / s_{e}^{4}$ a constant function of $\theta_{e}$. This choice ensures that the recorded experimental coherent atomic scattering for a monatomic gas has the form

$$
\begin{aligned}
I_{\mathrm{at}}^{\mathrm{coh}} & =K^{\prime} s_{e}^{4}|f|^{2} \\
& \approx K^{\prime \prime}\left(Z^{2}-2 Z F\left(s_{e}\right)+F\left(s_{e}\right)^{2}\right)
\end{aligned}
$$

in which the central term $-2 Z F\left(s_{e}\right)$ contributes a projected electronic density when optically Fourier transformed. ${ }^{7}$

In studies from which the molecular information embodied in the projected internuclear density $\rho_{i f}^{P}$ is desired, a different filter function $\Phi$ is needed for optimum results. The best filter makes $I_{a t}\left(s_{e}\right)$ a constant function of impact parameter on the holographic plate $H_{e}$, thereby making the intensity incident on $H_{e}$ proportional to $\left[1+M\left(s_{e}\right)\right]$. Recognizable internuclear peaks can be obtained with much simpler filters, however, as will be shown in the following paper. ${ }^{11}$

\section{Image formation}

First, we shall derive the form of the molecular information recorded on the holographic record, $H_{1}$, il- 
lustrated in Fig. 2. From this we shall then show how the intensity of the reconstruction is related to the structure of the molecule.

A photographic record of the electron intensity received at $H_{e}$ could, in principle, be used directly as a hologram. Optical imperfections in standard electron image plates make it desirable to copy the hologram, reducing it substantially in size, onto high quality holographic plates to get hologram $H_{1}$. Responses of photographic emulsions to electrons and to light are quite different. Fast electrons produce a photographic absorbance $A_{e}=-\log T_{e}$ in the developed plate $H_{e}$, where $A_{e}$ is a nearly linear function of the exposure. ${ }^{14}$ For the present purposes it is sufficient to assume that $A_{e}\left(b_{e}\right)$ is proportional to $I\left(b_{e}\right)$ of Eq. (3). In making the photographic reduction $H_{1}$, the light incident upon $H_{1}$ is proportional to the fraction, $T_{e}$, of the light transmitted through $H_{e}$. The associated absorbance, $A_{1}=-\log T_{1}$, in the developed plate $H_{1}$, may be assumed to follow the Hurter and Driffield photoresponse law ${ }^{15}$

$$
\begin{aligned}
A_{1} & =C^{\prime}+\Gamma \log E \\
& =\Gamma\left(\log C^{\prime \prime}+\log T_{e}\right),
\end{aligned}
$$

where $C^{\prime \prime}$ and $C^{\prime}$ are constants, $\Gamma$ is a contrast parameter characteristic of the plate and its development, and $E$ is an exposure proportional to $T_{e}$. Taking all of the foregoing into account, we find that the fraction of light, $T_{1}\left(b_{1}\right)$, transmitted through the developed plate $H_{1}$ at impact parameter $b_{1}$, is

$$
T_{1}\left(b_{1}\right)=C^{2} \exp \left(2.303 \Gamma A_{e}\right) \text {, }
$$

where $C^{2}=\left(C^{\prime \prime}\right)^{-\Gamma}$. By absorbing various proportionality constants into a new constant $K$, we can rewrite Eq. (10) as

$$
T_{1}\left(b_{1}\right)=C^{2} \exp \left[2 K I\left(b_{e}\right)\right],
$$

where $b_{e}$ maps into $b_{1}$ by whatever reduction factor is used in copying $H_{e}$.

Now let us calculate the Fraunhofer diffraction pattern (the image) obtained by passing a monochromatic plane wave of light through hologram $H_{1}$. Let us take for $U\left(b_{1}\right)$, the amplitude of the plane wave transmitted through the hologram, the square root of the transmission of Eq. (11), or

$$
\begin{aligned}
U\left(b_{1}\right) & =C \exp \left[K I\left(b_{e}\right)\right] \\
& =C \exp \left[K I_{\mathrm{at}}\left(1+M\left(s_{e}\right)\right)\right] \\
& =C\left[\exp K I_{\mathrm{at}}\right]\left[\exp K I_{\mathrm{at}} M\left(s_{e}\right)\right] \\
& \approx C\left[\exp K I_{\mathrm{at}}\right]\left[1+K I_{\mathbf{2 t}} M\left(s_{e}\right)+\cdots\right] \\
& =U_{0}\left(b_{1}\right)+U_{m}\left(b_{1}\right) .
\end{aligned}
$$

If the hologram $H_{e}$ is not too dark, the molecular amplitude $U_{m}\left(b_{1}\right)$ is adequately represented by the linear expansion term. In any case, the amplitude of light diffracted by $H_{1}$ as a function of scattering angle $\theta_{2}$ is given, in the small angle range of interest, by

$$
\begin{aligned}
F\left(s_{2}\right) & =\int \exp \left(\mathrm{s}_{2} \cdot \mathrm{b}_{1}\right) U\left(b_{1}\right) d^{2} b_{1} \\
& =F_{0}+F_{m},
\end{aligned}
$$

where $s_{2}$, the difference between incident and scattered wave vectors, has a magnitude $s_{2}=\left(4 \pi / \lambda_{2}\right) \sin \left(\theta_{2} / 2\right)$. Since $U\left(b_{1}\right)$ is the sum of a leading term and a molecular term, so also is $F\left(s_{2}\right)$, as shown above. If the filter function $\Phi$ makes $I_{\text {at }}$ constant, then $U_{0}\left(b_{1}\right)$ is constant out to the maximum value of $b_{1}$ and $F_{0}$ is a strong Airy diffraction pattern whose central disc radius is the defined Abbe resolution limit of the "micrograph" generated and whose outer rings may seriously interfere with the desired image unless filtered out as discussed in the next section.

A relationship between the molecular amplitude $F_{m}$ and the molecular structure is developed as follows. Equation (13), with $U_{m}$ given by Eq. (12), can be integrated numerically without recourse to further approximation to obtain a theoretical representation to be compared with experiment. Curves so calculated are presented in the following paper. ${ }^{11}$ A more direct appreciation of the significance of $F_{m}$ can be derived in the case of holograms obtained under optimum conditions. Such conditions imply lightly exposed holograms gener ated with a filter function $\Phi$ designed to make $I_{\text {at }}$ a constant, and a filtering out of incoherent scattering [the $S_{k}$ of Eq. (6) plus the adoption of atoms sufficiently low in atomic number that the coefficients $A_{i f}$ in the reduced intensity function are nearly constant functions of $s_{e}$. When these conditions are satisfied, and Eqs. (4)-(8), (12), and (13) are combined, the molecular amplitude $F_{m}\left(s_{2}\right)$ becomes

$$
\begin{aligned}
F_{m}\left(s_{2}\right) \approx & K_{m}^{\prime} \iint \exp \left(i \mathbf{s}_{2} \cdot \mathrm{b}_{1}\right) \\
& \times \exp \left(i \mathrm{~s}_{e} \cdot \mathrm{b}_{a}\right) \rho^{P}\left(b_{a}\right) d^{2} b_{1} d^{2} b_{a},
\end{aligned}
$$

where $K_{m}^{\prime}=C K I_{\mathrm{at}} \exp \left(K I_{\mathrm{at}}\right)$ and where

$$
\rho^{P}\left(b_{a}\right) \equiv 2 \sum_{i<j} \sum_{i j} \rho_{i j}^{P}\left(b_{a}\right) .
$$

The expression becomes more transparent when changes of variable appropriate for modest scattering angles are made, namely,

$$
\mathrm{b}_{1} \approx\left(L_{e} \lambda_{e} / 2 \pi R\right) \mathbf{s}_{e}
$$

and

$$
\begin{aligned}
\mathrm{b}_{2} & \approx\left(L_{2} \lambda_{2} / 2 \pi\right) \mathbf{s}_{2} \\
& \equiv\left(R L_{2} \lambda_{2} / L_{e} \lambda_{e}\right) \mathrm{b}_{a}^{\prime},
\end{aligned}
$$

where $R$ is the factor by which the hologram $H_{e}$ was reduced in forming hologram $H_{1}$, and where the new vector $b_{a}^{\prime}$ is defined. Upon substitution into Eq. (14) we find

$F_{m}=K_{m} \iint \exp \left(i \mathrm{~b}_{a}^{\prime} \cdot \mathbf{s}_{e}\right) \exp \left(i \mathbf{s}_{e} \cdot b_{a}\right) \rho^{P}\left(b_{a}\right) d^{2} b_{d} d^{2} s_{e}$.

If the range of $\mathbf{s}_{e}$ were so great that the limits could be taken as from minus infinity to infinity (the limits of $b_{a}$ are this great) we could invoke the Fourier identity

$$
\begin{aligned}
\rho^{P}\left(\mathrm{~b}_{a}^{\prime}\right)= & \left(1 / 4 \pi^{2}\right) \int_{-\infty}^{\infty} \int_{-\infty}^{\infty} \exp \left(i \mathrm{~b}_{a}^{\prime} \cdot \mathrm{s}_{e}\right) \\
& \times \exp \left(i \mathrm{~s}_{e} \cdot \mathrm{b}_{a}\right) \rho^{P}\left(b_{a}\right) d^{2} b_{a} d^{2} s_{e}
\end{aligned}
$$

and make the identification 


$$
F_{m} \propto \rho^{P}\left(b_{a}^{\prime}\right),
$$

so that the image amplitude would turn out to be just the molecular projected density discussed in Sec. II A but enlarged by the enormous factor $R L_{2} \lambda_{2} / L_{e} \lambda_{e}$. Note that $\lambda_{2} / \lambda_{3}$ by itself is characteristically $6000 \AA / 0.06 \AA=10^{5}$. Subsequent enlargement by an eyepiece or projector lens can, together with the factor $R L_{2} / L_{e}$, easily increase the overall magnification to a value greater than $10^{8}$.

It must be recalled that the image intensity, as seen or photographed through the second stage eyepiece, corresponds to the square of the amplitude $F_{0}+F_{m}$. Implicit in the above expressions is the fact that the characteristic twin images of holography are obtained. In the present case in which the images naturally have a center of symmetry, the twin images are identical. Since they are superposed on each other they are not seen separately.

In what sense can the Fraunhofer amplitude $F_{m}$ be interpreted as a true molecular image, even if it is proportional to the projected density $\rho^{P}\left(b_{a}^{\prime}\right)$ in accord with Eq. (20)? The answer is that when the conventional holographic condition is satisfied, the image $\rho^{p}\left(b_{a}^{\prime}\right)$ takes on a simple meaning. That is, when there is a single dominant reference beam generated in each molecule, by scattering from a single atom, $n$, of substantially greater scattering power than the others, then the expression $\rho^{P}\left(b_{a}\right)$ of Eq. (15) simplifies. Only those coefficients $A_{i j}$ with $i$ or $j$ equal to $n$ will contribute heavily and $\rho^{P}\left(b_{a}\right)$ reduces to

$$
\rho^{P}\left(b_{a}\right) \approx 2 \sum_{i} A_{\mathrm{in}} \rho_{\mathrm{in}}^{P}\left(b_{a}\right) .
$$

This function exhibits maxima at radii associated with all the internuclear distances between the dominant atom $n$ and the others, and corresponds exactly to the rota tional average image of the molecule pivoting about its heavy atom. The subsidiary peaks with coefficients $A_{i f}$ not referring to atom $n$ correspond to minor image distortions encountered also in conventional holography from the square of the "subject-wave" amplitude. Quite apart from any reference to the theory of image formation, it is apparent that the Fraunhofer diffraction pattern generated by passing a plane wave through a suit ably filtered electron diffraction pattern should manifest the spectrum of internuclear distances in the molecule. Indeed, it may be possible to recover more structural information about molecular structure when there are several strong scatterers in the molecule than when there is only one.

A serious impediment to the observation of the projected density function $\rho^{P}\left(b_{a}\right)$ embodied in $F_{m}$ is the strong amplitude $F_{0}$ naturally accompanying $F_{m}$ as shown in Eq. (13). In conventional optical Fourier transform holography this presents no difficulty be cause $F_{0}$ is a focused spot so fine that it does not overlap the image. In the present situation, however, the molecular images lie so close to the central beam focus that the inner shells of $\rho^{P}\left(b_{a}\right)$ begin by about the sixth to tenth Airy diffraction ring of $F_{0}$. Since the image intensity from a lightly recorded hologram may be far less than the intensity of the inner Airy rings, the image may be seriously obscured. Therefore, it is essential to suppress the Airy pattern. Methods to accomplish this are discussed in the next section.

\section{Suppression of spurious Airy pattern}

In principle it is possible to null out the unwanted amplitude $U_{0}$ of $\mathrm{Eq}$. (12), by destructive interference, before it can propagate on to the Fraunhofer pattern. Furthermore, this suppression can be accomplished without significantly altering the desired amplitude $U_{m}$. Such a filtering could be done by placing $H_{1}$ in one leg of a Mach-Zehnder interferometer, inserting a comparison hologram $H_{1}^{\prime}$ in the other leg, and adjusting the relative phases as discussed in Ref. 7. If $H_{1}$ were taken with a polyatomic gas and $H_{1}^{\prime}$ with a monatomic gas, the $U_{0}\left(b_{1}\right)$ functions of the two holograms could be made sufficiently similar to lead to the annihilation of the outgoing $U_{0}$ term. Since $H_{1}^{\prime}$ would possess no molecular features, it could not disturb the molecular term $U_{m}$, and the Fraunhofer patter of Eq. (13) would be just the molecular contribution $F_{m}$.

In practice such an interferometric filtering would be extremely difficult. Therefore, an alternative "spatial domain filter" was devised, following a suggestion by Professor Emmett Leith. ${ }^{16}$ It greatly reduces the unwanted signal by a judiciously placed stop, projector lens, and diaphragm, as illustrated in Paper II. ${ }^{11}$

\section{DISCUSSION}

We have demonstrated that a variant of holography can, in theory, be carried out with electron waves in such a way as to achieve a resolving power of the or der of $10^{-1} \AA$, and render visible interatomic distances in molecules. The generation of the reference wave, internally within the molecules investigated instead of externally, maximizes holographic fringe separations and minimizes aberrations and requirements for coherence and for mechanical stability of the system. Coherence lengths in the first stage scarcely need to be much more than molecular lengths. In the second stage, as well, temporal coherence requirements are low. Partly balancing these advantages is the inherent disadvantage that, for our gaseous specimens, only rotational average images are produced, and these are averaged over an ensemble of molecules. Single molecules are not seen. Of course, to render single molecules visible whether holographically or otherwise, would be to subject them to severe radiation damage. In the present method there is no such necessity. Each molecule in the ensemble studied need experience only a single encounter with the incident quanta and the reconstruction, in consequence, can be shown to pertain to molecules in their initial, electronically unexcited, states.

$X$ rays could, in principle, be used instead of electrons in the first stage of the procedure. They would suffer several disadvantages, however. In the first place it is not a simple matter to record high quality $x$-ray patterns from gaseous samples, though it can be 
done. ${ }^{17}$ Secondly, conveniently available $x$-ray wavelengths are 1 or 2 order $s$ of magnitude longer than convenient electron wavelengths, and such long wavelengths would give much poorer resolving power. Even if short wavelengths were adopted, however, incoherent scattering makes a far greater contribution to $\mathrm{x}$-ray patterns at moderate to large $s$ values than to electron patterns, and this would worsen the signal to noise problem.

If a sample of randomly spaced but perfectly oriented molecules were available, the present approach would yield impressive images of molecules, unspoiled by rotational smearing. That possibility is remote and impossible in the gaseous phase. What is possible, in principle, is less spectacular, perhaps, but is nevertheless worthy of serious consideration. Whether the approach is feasible in practice is assessed in the following paper. ${ }^{11}$

\section{ACKNOWLEDGMENT}

This research was supported by a grant from the $\mathrm{Na}$ tional Science Foundation.

${ }^{1}$ H. Mark and R. Wierl, Naturwissenschaften 18, 205 (1930).
${ }^{2}$ D. Gabor, Nature (London) 161, 777 (1948); Proc. R. Soc. London Ser. A 197, 454 (1949).

${ }^{3}$ W. L. Bragg, Z. Kristallogr. 70, 475, 483 (1929); Nature (London) 143, 678 (1939); ibid. 149, 470 (1942).

${ }^{4}$ M. J. Buerger, Proc. Nat1. Acad. Sci. U. S. 27, 117 (1941); ibid. 36, 330 (1950); J. Appl. Phys. 21, 909 (1950).

${ }^{5} \mathrm{E}$. Abbe, Arch. Mikrosk. Anat. 9 (1973).

${ }^{6}$ L. S. Bartell and C. L. Ritz, Science 185, 1163 (1974).

${ }^{7}$ L. S. Bartell, Optik 43, 373, 403 (1975).

${ }^{8}$ L. S. Bartell and R. D. Johnson, Nature (London) 268, 707 (1977).

${ }^{9} \mathrm{G}$. S, Stroke, An Introduction to Coherent Optics and Holography (Academic, New York, 1966).

${ }^{10}$ A. L. Patterson, Phys. Rev. 46, 372 (1934); Z. Kristallogr. 90, 517 (1935).

${ }^{11}$ L. S. Bartell and W. J. Gignac, J. Chem. Phys. 70, 3958 (1979), following paper.

${ }^{12}$ T. C. Wong and L. S. Bartell, J. Chem. Phys. 58, 5654 (1973).

${ }^{13}$ R. J. Glauber, Lectures in Theoretical Physics, edited by W. E. Brittin and L. G. Dunham (Interscience, New York, 1959), Vol. 1.

${ }^{14}$ L. S. Bartell and L. O. Brockway, J. Appl. Phys. 24, 656 (1953).

${ }^{15}$ See R. J. Collier, C. B. Burkhardt, and L. H. Lin, Optical Holography (Academic, New York, 1971), Chap. 2.

${ }^{16} \mathrm{E}$. N. Leith, Photogr. Sci. Eng. 6, 75 (1962).

${ }^{17}$ M. H. Pirenne, The Diffraction of X-rays and Electrons by Free Molecules (Cambridge U. P., London, 1946). 